\title{
ADAPTIVE WING CAMBER OPTIMIZATION: A PERIODIC PERTURBATION APPROACH
}

\author{
MARTÍN ESPAÑA ${ }^{\dagger}$ and GLENN GILYARD \\ NASA Dryden Flight Research Center, P. O. Box 273, Edwards, California 93523, USA
}

\begin{abstract}
Available redundancy among aircraft control surfaces allows for effective wing camber modifications. As shown in the past, this fact can be used to improve aircraft performance. To date, however, algorithm developments for in-flight camber optimization have been limited. This paper presents a perturbational approach for cruise optimization through in-flight camber adaptation. The method uses, as a performance index, an indirect measurement of the instantaneous net thrust. As such, the actual performance improvement comes from the integrated effects of airframe and engine. The algorithm, whose design and robustness properties are discussed, is demonstrated on the NASA Dryden B-720 flight simulator.
\end{abstract}

Key words. Adaptive optimization; variable camber control; drag optimization; transport aircraft; adaptive control

\section{INTRODUCTION}

Because of increasing competition among airline manufacturers and operators, the world has recently seen an all-out effort to reduce airline direct operating costs (DOCs). Given that an airline's net profit is a percentage based on the difference between two large numbers (revenues and costs), a small drop in costs has considerable leverage in an industry with annual profits of just about 5 percent (National Research Council, 1992). Besides ownership ( $\sim 50$ percent of DOCs) the second major driver of the DOCs of an airline is fuel consumption, which accounts for around 18 percent. This shows the impact of aircraft performance on an operator's profitability, particularly because the operator does not usually control production costs.

In the late 1970's and 1980's, many research activities were conducted to improve performance aircraft as part of NASA programs on aircraft energy efficiency and advanced fighter technology integration. (Gilyard and España (1994) surveys past attempts to apply active controls to improve aircraft performance.) The F-111 mission adaptive wing program (Phillips and Smith, 1989) showed the real potential of the variable-wing-camber concept to improve aircraft performance. Two modes of the F-111 mission

${ }^{\dagger}$ This author holds a National Research Council-NASA Dryden Research Associateship. adaptive wing are of significant interest: (1) the cruise camber control mode, designed to use realtime adaptive optimization for drag reduction; and (2) the maneuver camber control mode, designed to maximize lift-drag ratio. The concept of both modes was successfully demonstrated in flight, though each mode has methodological limitations in practical implementation. The maneuver camber control mode requires accurate models stored in table lookup form onboard the aircraft. The cruise camber control mode has an optimization algorithm that is not suited for the low levels of drag improvements available on transport aircraft.

More recently, extensive wind-tunnel testing and flight experiments with wide-body transport, performed in Germany at the MBB Company (a member of the European consortium, Airbus Industrie), show that continuous camber variations can improve the efficiency of even the most advanced wings at their best design point (Renken, 1988). The reason is that the wing design is the result of a compromise between all possible flight conditions. The same team shows that coordinated deflections of flaps, ailerons, elevators, horizontal stabilizer, and possibly leading-edge devices can be used to induce variable wing cambering without the penalty of added weight or new wing box design (Renken, 1985; Renken, 1988; Szodruch, 1985). Besides drag improvements (potential increase of 3 to 9 percent in lift-drag ratio 
(Renken, 1988)) camber control may also have a positive impact on other aspects of the aircraft design, such as buffet limit (12-percent increase (Renken, 1985; Renken, 1988)) or root bending moments (Szodruch, 1985). The multiple benefits of the technology have recently prompted the highest level of the Airbus consortium to declare that "... variable cambering wing features will be mandatory for future megatransports" (New Large Aircraft, 1993).

The recent introduction of digital fly-by-wire technology in modern transport aircraft considerably eases the implementation of wing camber optimization techniques. To date, however, algorithm developments for in-flight adaptive camber optimization have been limited. As such, the development of a robust and efficient algorithm to adjust, in flight, the aircraft control surfaces configuration is in order. This paper considers a perturbational approach for cruise optimization through in-flight wing camber adaptation. The method is based on the measurement of a performance index that includes the engine contributions. The actual performance improvement comes, thus, from the integrated effects of airframe and engine. The algorithm is demonstrated on the B-720 flight simulator of NASA Dryden Flight Research Center.

Symmetric outboard and inboard ailerons, flaps, elevator, and horizontal stabilizer are possible surfaces available for optimization. The concept is illustrated here using symmetric outboard ailerons to produce effective wing camber changes together with the horizontal stabilizer for pitch-moment compensation. This paper addresses the analysis and design aspects of the algorithm. Research is currently in progress to extend these results to multisurface optimization. This extension could easily include the integration of decision variables from both the airframe and the engine.

\section{PROBLEM FORMULATION}

Adaptive optimization techniques with periodic perturbation and direct feedback of a measurable performance index (PI) allow for direct performance optimization without requiring a model (Eveleigh, 1967; Draper and Li, 1951). In this paper, use is made of the acceleration (as a net thrust measurement) and velocity as indexes. The flight regime considered is cruise with fixed power lever angle (PLA) position. Similar principles can be applied to optimize range or fuel flow (Gilyard and España, 1994). The in-flight optimization of other flight regimes, such as uniform climbing, could also be envisioned. The algorithm's viability is demonstrated using a single decision variable chosen to be symmetric deflection of the outboard ailerons $\left(\delta_{a i l}\right)$. The flight altitude, seen as an optimization constraint, is held by the autopilot, which sends commands to the elevator $\left(\delta_{e}\right)$ and the horizontal stabilizer $\left(\delta_{s t b}\right)$.

From the longitudinal flight equations the horizontal acceleration component, $a_{x}$, is given by

$$
\begin{aligned}
\mathrm{PI}= & a_{x}=\frac{1}{m}[T(F C) \cos \alpha- \\
& \left.\bar{q}(V, \rho) S C_{D}\left(\delta_{a i l}, F C\right)-m g \sin \gamma\right] \cos \gamma
\end{aligned}
$$

where $\alpha$ is the angle of attack, $m$ is the mass of the aircraft, $\bar{q}$ is the dynamic pressure, $V$ is the airspeed, $\rho$ is the air density, $S$ is the wing area, and $C_{D}$ is the drag coefficient. $F C$ stands for flying conditions and includes all unknown or unmodeled effects of those changing conditions, such as weight, center-ofgravity position, winds, velocity, altitude, aircraft aging, etc. $T(F C)$ corresponds to the engine static characteristics, relating thrust $(T)$ with $F C$. The surface deflections $\delta_{e}$ and $\delta_{s t b}$ do not appear explicitly in Eq. (1) because these values are implicitly determined by the autopilot for a given $\delta_{a i l}$ deflection. Under (perfect) cruise conditions (i.e., $\gamma=\dot{\gamma}=0$, where $\gamma$ is the path angle) $a_{x}=0$, while the total derivative,

$$
\begin{aligned}
\frac{d a_{x}}{d \delta_{a i l}}=\frac{1}{m}[ & \frac{d}{d \delta_{a i l}} T(F C) \cos \alpha- \\
& \left.\quad \bar{q}(V, \rho) S \frac{d}{d \delta_{a i l}} C_{D}\left(\delta_{a i l}, \mathrm{FC}\right)\right]
\end{aligned}
$$

is equal to zero at the optimal aileron deflection $\delta_{\text {ail }}^{*}$ (i.e., for $d a_{x l}\left(\delta_{c i l}^{*}\right) / d \delta_{a i l}=0$ ), which corresponds to an extremum of the acceleration, here used as the PI. Notice that the optimization naturally takes into account engine thrust changes caused by velocity changes. Figure 1 shows the autopilot and the optimizer loops, where $h, \dot{h}$, and $h_{D}$ are, respectively, the altitude, its time-derivative, and the engaged altitude.

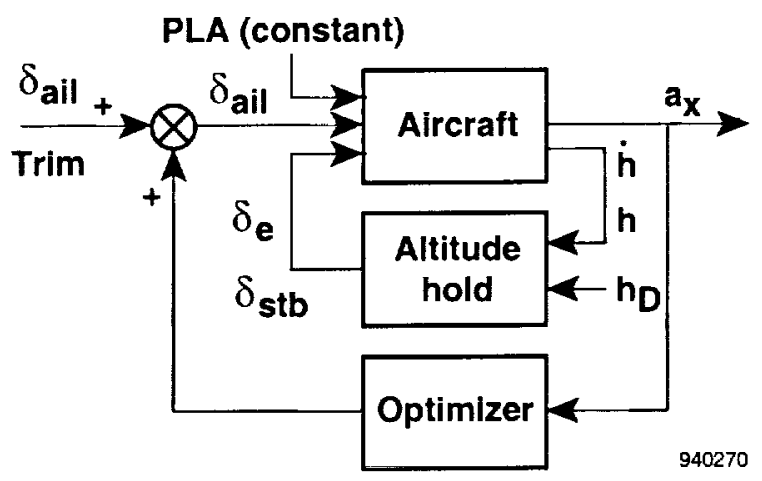

Fig. 1. Control diagram with autopilot and optimizer loops. 


\section{A PERIODIC PERTURBATION EXTREMA- SEARCHING TECHNIQUE: THE WORKING PRINCIPLE}

The method, first proposed by Draper and Li (1951), uses sinusoidal perturbations on each of the decision variables of the optimization problem and correlates (in a sense that will become clearer later) those signals with the measured PI. The vector of correlations is proportional to the local gradient of the PI, thus giving the best direction in the parameters space to improve the PI value. The basic principle of the algorithm is best described for a quadratic PI, here indicated by $J$. For constants $K, \Gamma>0$ (where $\Gamma$ is the curvature of PI in the scalar case), let

$$
\begin{aligned}
J(\delta) & =J\left(\delta^{*}\right)+\frac{1}{2} \Gamma\left(\delta-\delta^{*}\right) \\
\delta(t) & =\delta_{r}(t)+A \sin \left(\omega_{0} t\right) \\
\dot{\delta}_{r} & =-K J(\delta(t)) A \sin \left(\omega_{0} t-\varphi\right) ; \\
\delta_{r}(0) & =\delta_{0}
\end{aligned}
$$

where $\delta$ is the decision variable with initial value $\delta_{0}$ and optimal value $\delta^{*}, \delta_{c}$ is the control input, $\omega_{0}$ is the perturbation signal frequency, and $\varphi$ is an extra degree of freedom used later for design purposes. Given its nonlinear character, an exact analytic description of Eqs. (3) to (5) is a complicated task. An approximated analysis is based on the assumption that $\delta_{c}(t)$ changes much more slowly than the sinusoidal probing signal (and $J(t)$ ). This assumption is ensured with a sufficiently small integration gain $K$. The approximated technique (see, for instance, chap. 6 in Astrom and Witenmark, 1989) consists of substituting the right-hand side of Eq. (5) by its time average over the time interval $T_{0}=2 \pi / \omega_{0}$, which, for a function $f(t)$, is defined as

$$
\bar{f}(t)=\operatorname{avg}\{f(t)\}:=\frac{1}{T_{0}} \int_{t-T_{0}}^{t} f(\tau) d \tau
$$

where $\tau$ is the integration variable. The following approximation of expression (3), valid for small amplitudes $A$, is used:

$$
\begin{aligned}
J\left(A \sin \left(\omega_{0} t\right)\right. & \left.+\delta_{c}(t)\right) \cong J\left(\delta_{c}(t)\right) \\
& +\Gamma\left(\delta_{c}(t)-\delta^{*}\right) A \sin \left(\omega_{0} t\right)
\end{aligned}
$$

Using Eq. (7) and definition (6), and assuming that $\delta_{c}(t)$ is constant in $T_{0}$, the averaged right-hand side of Eq. (5) is calculated as

$$
\begin{aligned}
& K \Gamma\left(\delta_{c}(t)-\delta^{*}\right) A^{2} \text { avg }\left\{\sin \left(\omega_{0} t\right) \sin \left(\omega_{0} t-\varphi\right)\right\}= \\
& \frac{1}{2} K \Gamma\left(\delta_{c}(t)-\delta^{*}\right) A^{2} \text { avg }\left\{\cos (\varphi)-\cos \left(2 \omega_{0} t-\varphi\right)\right\}
\end{aligned}
$$

From Eq. (8) the averaged version of the nonlinear differential Eq. (5) is

$$
\dot{\bar{\delta}}_{c} \equiv-\frac{1}{2} K \Gamma\left(\bar{\delta}_{c}-\delta^{*}\right) A^{2} \cos (\varphi)
$$

Notice that, though the original Eq. (5) is a nonlinear time-varying differential equation, its averaged solutions $\bar{\delta}_{c}$, obey a linear differential equation. Whenever $\varphi \in(-\pi / 2, \pi / 2), \bar{\delta}_{c}$ converges exponentially to the optimum value $\delta^{*}$ with time constant $2 / K A^{2}$ $\Gamma \cos (\varphi)$. Important properties can be derived from the above analysis-

(P1) In the average $\delta_{r}$ tends exponentially to its optimal value $\delta^{*}$ for wide ranges of $\varphi, K$, and $\Gamma$.

(P2) Biases on the measurements do not affect this result because, from Eqs. (6) to (8), their averaged effect on Eq. (9) is zero.

\section{PRACTICAL IMPLEMENTATION OF THE ALGORITHM: EQUIVALENT CIRCUIT AND DESIGN}

Figure 2 shows a block diagram of a practical single-dimensional extremum-searching system. The plant's PI measurement process is represented by a nonlinear characteristic $J(\cdot)$ in series with a linear filter $G_{p}(\mathrm{~s})$ representing possible sensor dynamics. $G_{f}$ and $G_{a}$ are, respectively, a signal-shaping filter used to eliminate undesired frequency components at either side of $\omega_{0}$, and a low-pass filter. All transfer functions are assumed to have unitary gains. The tan$\operatorname{dem}\left(M, G_{a}\right)$ in Fig. 2 acts as a demodulator, eliminating most of the $\omega_{0}$ harmonics remaining in the feedback loop (mainly the $2 \omega_{0}$ term; see Eq. (8)) . As an exponentially weighted time average of $\pi$, the output of $G_{a}$, is an estimate of the current correlation of the inputs to the multiplier $M$. This correlation interpretation of the algorithm and the fact that sinusoidal signals of different frequencies have zero correlation explains a third important property of the algorithm, i.e.,

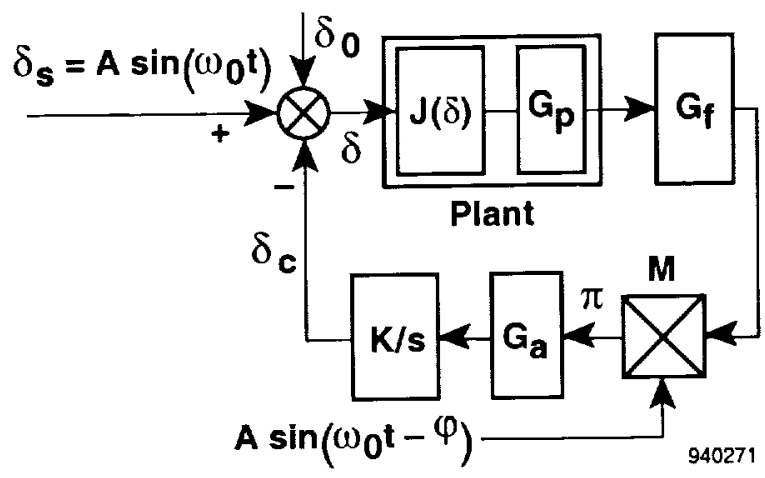

Fig. 2. A single-dimensional extrenum-searching algorithm. 
(P3) The effects of measurement noise on PI are negligible unless its power spectrum is concentrated on the excitation frequency $\omega_{0}$. (This may impose conditions on the choice of the amplitude $A$, the frequency $\omega_{0}$, or both.)

Property (P3) is enhanced in practice by using a small band pass filter centered on the excitation frequency $\omega_{0}$. Let $\varphi_{0}$ be the phase angle introduced by the cascaded block $G_{f} G_{p}(s)$ at $\omega_{0}, \varphi$ is used to compensate for this phase shift. The term $\delta_{0}$ is the trim value at the time of the algorithm engagement.

For design and analysis purposes, only the information contained in the low-frequency components of the signals in the circuit is of interest. For that reason, the system in Fig. 2 is transformed to an equivalent low-pass network by following the guidelines of reference 8 , chapter 9 . Figure 3 shows the corresponding equivalent network for a quadratic $J$ as in Eq. (3). Under each of the blocks of Fig. 3 is indicated, in parenthesis, the originating block from Fig. 2. In the same figure, $\bar{\delta}_{c}$ represents the (averaged) low-frequency component of the feedback signal $\delta_{\text {c. }}$. The parameters of the equivalent system are calculated as (Eveleigh, 1967)

$$
\begin{array}{ll}
\beta_{p}=\left|G_{p}\left(j \omega_{0}\right)\right| ; & \tau_{p}=-\left.\frac{\partial \psi_{p}(\omega)}{\partial \omega}\right|_{\omega=\omega_{0}} \\
\beta_{f}=\left|G_{f}\left(j \omega_{0}\right)\right| ; & \tau_{f}=-\left.\frac{\partial \psi_{f}(\omega)}{\partial \omega}\right|_{\omega=\omega_{0}}
\end{array}
$$

where $\left|G\left(j \omega_{0}\right)\right|$ and $\psi\left(\omega_{0}\right)$ indicate, respectively, the modulus and the phase angle of the transfer function $G$ at $\omega_{0}$. The equivalent network in Fig. 3 is then used for the stability analysis and design of the adaptive optimization algorithm. The designer has at his disposal the gain $K$, the phase compensation $\varphi$, the perturbation signal amplitude $A$, and the frequency $\omega_{0}$. The transfer functions of the filters $G_{f}$ and $G_{a}$ can also be used to refine the design. From the system in

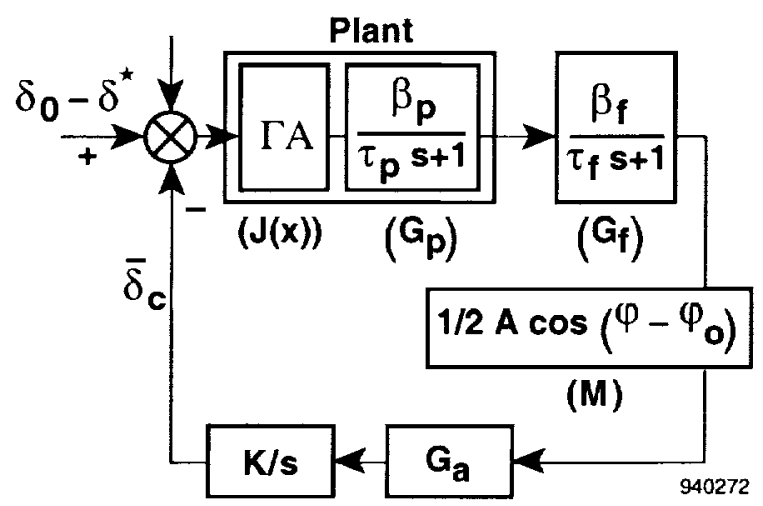

Fig. 3. Equivalent circuit of block diagram from Fig. 2.
Fig. 3, using standard linear techniques (e.g., root locus), one can easily conclude that for $K$ sufficiently small and $\tau_{p}>0, \tau_{f}>0$ (which is the case in practice), the integrator on the feedback path ensures the exponential convergence of $\bar{\delta}_{r}$ to its optimal value $\delta^{*}$, even under model inaccuracies. The convergence to the optimum is, thus, a robust property of the algorithm with respect to plant variations. As we saw, a small $K$ is also required for the validity of our analysis.

\section{APPLICATION TO ACTIVE CAMBER OPTIMIZATION}

The algorithm was tested using the NASA Dryden B-720 aircraft simulator at a nominal cruise flight condition $(30,000 \mathrm{ft}, 0.8 \mathrm{Mach}$, and total weight of $200,000 \mathrm{lb}$ ). The frequency $\omega_{0}=0.025 \mathrm{rad} / \mathrm{sec}$ is purposely made small in comparison with the bandwidth of the aircraft in closed loop with the autopilot. With this choice the autopilot ensures a good ride quality and diminishes the dynamic effects over the optimization constraints. With similar criteria an aileron deflection amplitude $A=2^{\circ}$ was considered acceptable.

\subsection{Design}

For the design, the sensor/aircraft dynamics (Fig. 2) are neglected by assuming $\beta_{p}=1$ and $\tau_{p}=0$. The trim point characteristic relating $\delta_{a i}$ with the PLA position (this curve can be determined experimentally in practice) was fitted with a second-order parabola used to calculate the characteristic's average curvature $\Gamma_{P L A}$. This value $\Gamma_{P L A}$ is then normalized by $\bar{q}=1 / 2 \rho \mathrm{V}^{2}\left(281 \mathrm{lb} / \mathrm{ft}^{2}\right)$, giving: $\bar{\Gamma}_{P L A}:=$ $\Gamma_{P L A} / \bar{q}=7.7 \mathrm{E}-04$. We then calculate the normalized curvature for the acceleration used as a PI as

$$
\bar{\Gamma}_{\mathrm{acc}}:=\mathrm{k}_{P L A} \bar{\Gamma}_{P L A} / m=2.85 \mathrm{E}-05
$$

where $k_{P L A}=230 \mathrm{lb} / \mathrm{deg}$ is the thrust-to-throttle gain evaluated from the engine data, and $m$ is the total aircraft mass. Notice that, given the convergence robustness discussed in the previous section, only approximated values of the above constants are required.

To keep the design simple, the following first-order unitary gain transfer functions were chosen for $G_{f}$ and $G_{a}$ :

$$
G_{f}=\frac{s}{s+\omega_{h}} ; \quad G_{a}=\frac{\omega_{l}}{s+\omega_{l}}
$$

Selecting $\omega_{h}=\omega_{l}=\omega_{0} / 2$ puts $\omega_{0}$ on the band pass of $G_{f}$ and out of the band pass of $G_{a}$. The phase angle induced by $G_{f}$ at the chosen $\omega_{0}$ is $\varphi_{f}=26.5^{\circ}$. Because the dynamics term $G_{p}$ has been neglected, one 
chooses, as shown in Figs. 1 and 2, $\varphi=\varphi_{0}=\varphi_{f}$ (the phase shift introduced by $G_{j}$ ). From Eq. (10) one has

$$
\begin{gathered}
\beta_{f}=\frac{\omega_{0}}{\left(\omega_{0}^{2}+\omega_{h}^{2}\right)^{1 / 2}}=2 / \sqrt{5} \\
\tau_{f}=\frac{\omega_{h}}{\left(\omega_{0}^{2}+\omega_{h}^{2}\right)^{1 / 2}}=\frac{2}{5 \omega_{0}}
\end{gathered}
$$

From Eqs. (12) and (13) the open-loop transfer function poles of the equivalent system in Fig. 3 are 0 , $\omega_{0} / 2$, and $5 \omega_{0} / 2$. After normalizing by $\omega_{0}$, the normalized open-loop transfer function results in

$$
\begin{gathered}
\frac{G / \omega_{0}^{3}}{s / \omega_{0}\left(s / \omega_{0}+1 / 2\right)\left(s / \omega_{0}+5 / 2\right)} \\
\bar{G}:=\frac{G}{\omega_{0}^{3}}=\frac{\sqrt{5} K \bar{q} \bar{\Gamma} A^{2}}{4 \omega_{0}}
\end{gathered}
$$

where $G$ is the combined gain of the blocks $(J),\left(G_{f}\right)$, $(M), G_{a}$, and $K / s$ of Fig. 3 put in cascade. The normalized dynamics of the performance optimization algorithm is characterized by the closed-loop poles of the transfer function (14). Those poles can be conveniently placed by an appropriate choice of the normalized gain $\bar{G}$. The critical normalized gain for a deadbeat response can be shown to be $\bar{G}_{c}=0.1408$, with a corresponding coincident dominant (and normalized) closed-loop poles at -0.24 and a single normalized fast pole at -2.52 . For the case under consideration with the average curvature $\bar{\Gamma}$ given by Eq. (11) and the selected $A$ and $\omega_{0}$, the design results in a pair of dominant poles at $-0.006 \mathrm{rad} / \mathrm{sec}$ and a fast pole at $-0.063 \mathrm{rad} / \mathrm{sec}$. The algorithm gain, $K=$ 0.196 is calculated using Eq. (14) as a function of the selected $\bar{G}$ and the rest of the design parameters. This completes the algorithm design. Equation (14) also gives an easy way to update $K$ with the dynamic pressure $\bar{q}$. Notice that for a given design criterion (in this paper we chose a critical design), the adaptation gain, the corresponding closed-loop poles, and, consequently, the algorithm convergence speed are all proportional to the excitation frequency $\omega_{0}$.

Given the small accelerations involved, the airspeed velocity may be a more suitable performance index than the acceleration itself. For this case the corresponding adaptation gain $K_{v e l}$ is calculated using the fact that the $\omega_{0}$-component of the measured signal $V_{x}$ satisfies

$$
a_{x}=\frac{d V_{x}}{d t} \Rightarrow V_{x}=V \cos \gamma=\frac{\alpha_{x}}{j \omega_{0}}
$$

Thus, to use $V_{x}$ as the measured PI, $K$ has to be modified by a factor of $\left|\omega_{0}\right|$ and the phase $\varphi$ delayed $90^{\circ}$. This gives $K_{v e l}=0.196 \omega_{0}$ and $\varphi=26.5^{\circ}-90^{\circ}=$ $-63.5^{\circ}$.

\subsection{Results}

Figure 3 shows the time histories of a simulated speed maximization flight experiment at $30,000 \mathrm{ft}$ and $0.8 \mathrm{Mach}$ (approximately $796 \mathrm{ft} / \mathrm{sec}$ ). The autopilot regulates the altitude while the PLA is kept at $62^{\circ}$ corresponding, approximately, to an engine
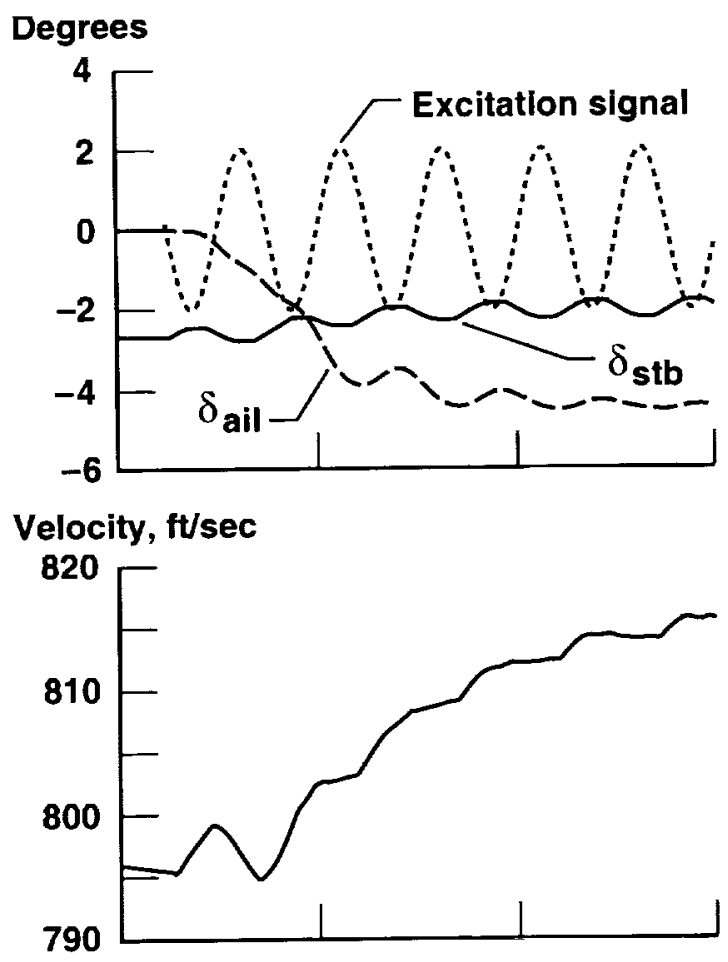

Altitude, $\mathrm{ft}$

$30.02 \times 10^{3}$

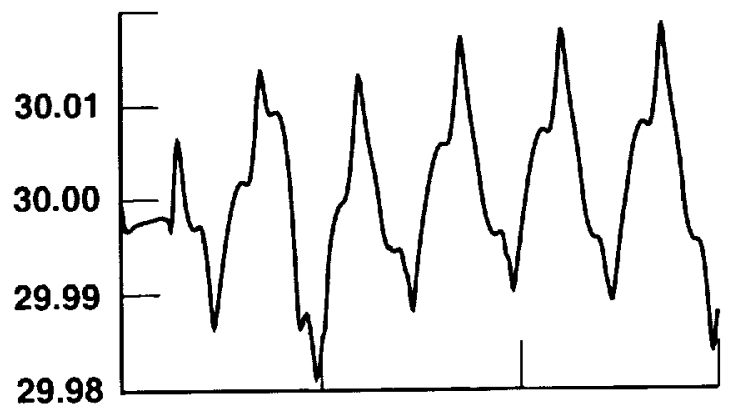

Normal acceleration, $\mathbf{g}$

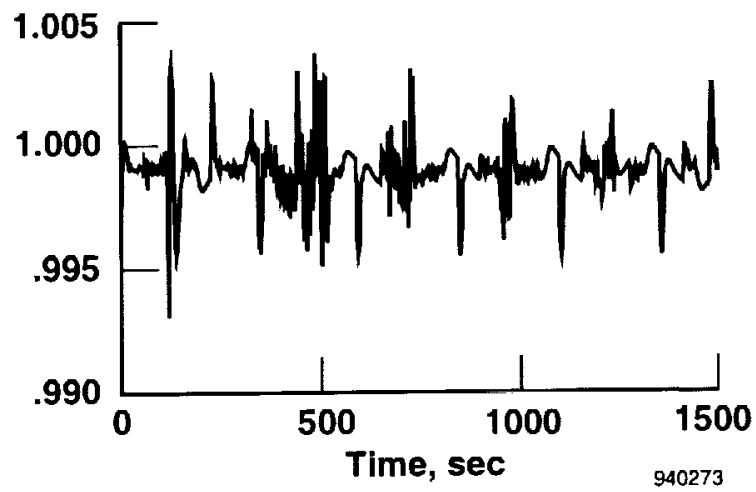

Fig. 4. Time histories from B-720 velocity optimization; Mach 0.8 at 30,000 ft; 200,000-lb gross weight. 
thrust level of $15,000 \mathrm{lb}$. The algorithm is engaged at $t=120 \mathrm{sec}$. Figure 4 displays the trim command $\left(\delta_{a i l}\right)$ sent to the aileron, the excitation signal superimposed on it, and the corresponding horizontal stabilizer deflection $\left(\delta_{s t b}\right)$ produced by the autopilot. Figure 4 also shows the actual speed time history indicating a speed increase of approximately 20 $\mathrm{ft} / \mathrm{sec}$. As seen in the figure, the periodic perturbation induces an altitude oscillation with the same fundamental period and an amplitude ranging from 10 to $20 \mathrm{ft}$. Finally, the control law is shown to have a minimal effect on the normal acceleration (less than $5 \mathrm{E}-03 \mathrm{~g}$ in absolute value) with negligible consequences on the ride quality.

\section{CONCLUDING REMARKS}

The possibility of continuously improving aircraft performance using conventional control surfaces for wing camber adaptation is demonstrated for a simulated transport aircraft. To take advantage of the full potential of the variable-camber concept, an algorithm based on a direct measurement of performance is required. In this paper a periodic perturbation technique is explored and its viability demonstrated. Besides its ease of implementation, the main advantages of the proposed technique are (1) its noise insensitivity and (2) its robustness with respect to measurement biases and model inaccuracies. Furthermore, the algorithm's design is simple and straightforward. Applying the algorithm to the velocity optimization problem results in a significant increase in cruise velocity for a simulated B-720 transport aircraft with in-flight adaptive repositioning of aileron/horizontal stabilizer deflections. The same algorithm can also optimize other performance indices such as range, acceleration (net thrust) or fuel flow. By using the proposed algorithm, performance improvement can be achieved for different flight conditions, weight changes, aircraft aging, and center-of-gravity position changes. The principle can be extended to multivariable optimization, which makes the algorithm suitable for application to airframe-propulsion integrated optimization. The fact that future aircraft will incorporate active control features in their aerodynamics and propulsion control effectors further sustains the significance of the results presented in this paper.

\section{REFERENCES}

Åström, Karl Johan and Björn Wittenmark (1989). Adaptive Control, Addison-Wesley Publishing Co., Reading, Massachusetts, pp. 235-246.

Draper, C. S. and Y. T. Li (1951). Principles of Optimizing Control Systems: an Application to the Internal Combustion Engine. Research Report, Aeronautical Engineering Department, Massachusetts Institute of Technology.

Eveleigh, V. W. (1967). Adaptive Control and Optimization Techniques. McGraw-Hill Book Co., New York.

Gilyard, Glenn B. and Martín D. España. (1994) On the Use of Controls for Subsonic Transport Improvement: Overview and Future Directions. AIAA-94-3515, Atmospheric Flight Mechanics Conference, Scottsdale, Arizona.

National Research Council (1992). Aeronautical Technologies for the Twenty-First Century. Committee on Aeronautical Technologie, Aeronautics and Space Engineering Board. National Academic Press, Washington, DC.

New Large Aircraft (1993). Flight International, pp. 34-36, Nov. 24-30, 1993.

Phillips, P. W. and S. B. Smith (1989). AFTLF-111 Mission Adaptive Wings (MAW) Automatic Flight Control System Modes Lift and Drag Characteristics. AFFTC-TR-89-03, U.S. Air Force Flight Test Center, Edwards AFB, California.

Renken, Jürgen (1985). Mission-Adaptive Wing Camber Control Systems for Transport Aircraft. AIAA-85-5006.

Renken, Jürgen (1988). Variable Wing Camber Control Systems for the Future Airbus Program, MBB-UT-104/88.

Szodruch, J. (1985). The Influence of Camber Variation on the Aerodynamic of Civil Transport Aircraft. AIAA-85-0353, Reno, Nevada. 


\section{Technical Presentation or Journal Article}

Paper title Transport Afrcraft Wing Camber Adaptive Optimization: A Pertodic Perturbation Approach

Author(s) Martin EspaAla and Glenn Gilyard (NASA Dryden Flight Research Center) Point of contact/phone Martin Espana (805) 258-3721

1] Meeting Conference Location Palo Alto, CA

Date Sept 12-16, Sponsor $/ F A C$ $\square$ Journal 1994 Preprint deadline 7-1-94 Paper no. Deadline (if applicable)

Classification: Unclassified $\mathbf{X}$ Confidential Secret Other

Distribution limitation: None FEDD ITAR Other Job order no. I $39701 \mathrm{~L}$ FF427 complete? zes Publish as a NASA report? No

\begin{tabular}{|c|c|c|c|c|}
\hline Approvals & Org. & Initial & Date & \multirow{4}{*}{$\begin{array}{l}\text { ATD USE ONLY } \\
\text { Date received } 5 / 6,94 \\
\text { H. } \frac{1998}{\text { Sue huke }} \\
\text { Editor Sue }\end{array}$} \\
\hline \multirow{3}{*}{$\begin{array}{r}\text { Branch } \\
\text { Division } \\
\text { Committee chair }\end{array}$} & $X E f$ & $Z, 1 / \& \in s$ & $3 / 10 / \pi 4$ & \\
\hline & $x k$ & lat & $3 / 22 / 94$ & \\
\hline & $S^{\prime} A L^{\prime}$ & $\rightarrow$ & $15-6-94$ & \\
\hline
\end{tabular}


Public reporting burden for this collection of information is estimated to average $f$ hour per response, including the time for reviewing instructions, searching existing dala sources. gathering and (this burden estimate or any other aspect

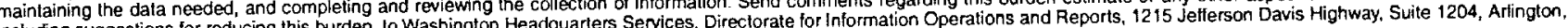
VA 22202-4302, and to the Office of Management and Budget, Paperwork Reduction Project (0704.0188), Washington, DC 20503.

\begin{tabular}{|l|l|l}
\hline 1. AGENCY USE ONLY (Leave blank) & $\begin{array}{l}\text { 2. REPORT DATE } \\
\text { September } 1994\end{array}$ & $\begin{array}{l}\text { 3. REPORT TYPE AND DATES COVERED } \\
\text { Conference Paper }\end{array}$ \\
\hline
\end{tabular}

4. TITLE AND SUBTITLE

Adaptive Wing Camber Optimization: A Periodic Perturbation Approach

\section{AUTHOR(S)}

Martin Espana and Glenn Gilyard

7. PERFORMING ORGANIZATION NAME(S) AND ADDRESS(ES)

8. PERFORMING ORGANIZATION

REPORT NUMBER

NASA Dryden Flight Research Center

P.O. Box 273

$\mathrm{H}-1998$

Edwards, California 93523-0273

10. SPONSORING/MONITORING

AGENCY REPORT NUMBER

National Aeronautics and Space Administration

Washington, DC 20546-0001

H-1998

11. SUPPLEMENTARY NOTES

Presented at the IFAC Symposium, Palo Alto, California, USA, 12-16 September 1994. Aerospace Control '94. Published in Automatic Control in Aerospace, (Aerospace Control '94), pp. 35-40.

12a. DISTRIBUTIONAVAILABILITY STATEMENT

12b. DISTRIBUTION CODE

Unclassified-Unlimited

Subject Category 02

\section{ABSTRACT (Maximum 200 words)}

Available redundancy among aircraft control surfaces allows for effective wing camber modifications. As shown in the past, this fact can be used to improve aircraft performance. To date, however, algorithm developments for in-flight camber optimization have been limited. This paper presents a perturbational approach for cruise optimization through in-flight camber adaptation. The method uses, as a performance index, an indirect measurement of the instantaneous net thrust. As such, the actual performance improvement comes from the integrated effects of airframe and engine. The algorithm, whose design and robustness properties are discussed, is demonstrated on the NASA Dryden B-720 flight simulator.

\begin{tabular}{l}
\hline 14. SUBJECT TERMS \\
Adaptive optimization, Variable camber control, \\
aircraft, Adaptive control. \\
\begin{tabular}{l|l} 
17. SECURITY CLASSIFICATION & 18. SECURITY CLASSIFICATION \\
OF REPORT & OF THIS PAGE \\
Unclassified & Unclassified \\
\hline
\end{tabular}
\end{tabular}

19. SECURITY CLASSIFICATION OF ABSTRACT

Unclassified
15. NUMBEA OF PAGES

6

16. PRICE CODE

$\mathrm{A} 02$

20. LIMITATION OF ABSTRACT

Unlimited 